\title{
3-卤-4-脂肪胺基-5-联苯氧基-2(5H)-呋喃酮的合成
}

\author{
叶 斌 ${ }^{a}$ 莫广珍 ${ }^{b, c}$ 关丽涛 ${ }^{d}$ 吴彦城 ${ }^{b}$ 陈任宏*,a 汪朝阳*,b \\ ( ${ }^{a}$ 广东食品药品职业学院 广州 510520) \\ ( ${ }^{b}$ 华南师范大学化学与环境学院 教育部环境理论化学重点实验室 广州 510006) \\ ( ${ }^{c}$ 佛山市顺德一中实验学校 顺德 528300) \\ ( $d$ 华南农业大学材料与能源学院 广州 510642)
}

\begin{abstract}
摘要 以硫酸为催化剂, 4-差基联苯与粘卤酸在甲苯溶剂中回流，经脱水醚化反应合成了 3,4-二卤-5-联苯氧基-2 $(5 H)$ 呋喃酮化合物. 以这 2 个新型的中间体与不同的代表性脂肪胺类在 KF 催化的室温条件下，通过串联的迈克尔加成-消 除反应，得到了 16 个新的 3-卤-4-脂肪胺基-5-联苯氧基- $2(5 H)$ 呋喃酮化合物，产率 43\% 79\%(大部分 55\%以上). 新化 合物的结构经 FTIR、UV、 ${ }^{1} \mathrm{H} N \mathrm{NM} 、{ }^{13} \mathrm{C} N \mathrm{~N} R 、 \mathrm{MS}$ 和元素分析确证.
\end{abstract}

关键词 2(5H)-呋喃酩; 联苯结构; 合成; 脂肪胺; 夰联迈克尔加成-消除反应

\section{Synthesis of 4-Amino-5-biphenyloxy-3-halo-2(5H)-furanones}

\author{
Ye, $\operatorname{Bin}^{a} \quad$ Mo, Guangzhen ${ }^{b, c}$ \\ Chen, Renhong*,a \\ Guan, Litao $^{d}$ \\ $\mathrm{Wu}$, Yancheng ${ }^{b}$ \\ Wang, Zhaoyang ${ }^{*, b}$ \\ ( ${ }^{a}$ Guangdong Food and Drug Vocational College, Guangzhou 510520) \\ $\left({ }^{b}\right.$ Key Laboratory of Theoretical Chemistry of Environment, Ministry of Education, School of Chemistry and \\ Environment, South China Normal University, Guangzhou 510006) \\ ( ${ }^{c}$ Shunde No. 1 Experimental Middle School, Shunde 528300) \\ $\left({ }^{d}\right.$ College of Materials and Energy, South China Agricultural University, Guangzhou 510642)
}

\begin{abstract}
Two novel 5-biphenyloxy-3,4-dihalo-2(5H)-furanones are synthesized via the direct dehydrative etherification reactions of biphenyl-4-ol with mucochloric acid and mucobromic acid, respectively, using sulfuric acid as a catalyst in toluene at reflux temperature. Taking them as intermediates, the tandem Michael addition-elimination reaction with different representative aliphatic amines is carried out in the presence of $\mathrm{KF}$ as a base and catalyst at room temperature. Sixteen 4-amino-5-biphenyloxy-3-halo-2(5H)-furanones are obtained, and the isolated yields are $43 \% \sim 79 \%$ (mostly over $55 \%$ ). The structures of all newly synthesized compounds were systematically characterized by FTIR, UV, ${ }^{1} \mathrm{H} \mathrm{NMR},{ }^{13} \mathrm{C}$ NMR, MS and elemental analysis. This investigation affords an important novel strategy for the synthesis of different $2(5 H)$-furanone derivatives having potential bioactivity, especially for the synthesis of $2(5 H)$-furanones simultaneously with biphenyl and amino structure.
\end{abstract}

Keywords 2(5H)-furanone; biphenyl structure; synthesis; aliphatic amine; tandem Michael addition-elimination reaction

2(5H)-呋喃酮结构单元广泛存在于天然产物 ${ }^{[1]}$ 与药 物分子 ${ }^{[2 \sim 4]}$ 中, 使 $2(5 H)$-呋喃酮化合物的合成在药物开 发中近来受到广泛关注 ${ }^{[5 \sim 8]}$. 许多联苯类化合物(图 1)具 有抗菌、抗癌等生物活性 ${ }^{[2,9]}$, 使联苯结构单元在药物分
子的设计与合成中最近也越来越受到重视 ${ }^{[10,11]}$. 但是, 目前仅有极少的文献报道将 2(5H)-呋喃酮结构与联苯 结构同时构建于潜在生物活性分子中的研究 ${ }^{[2,11]}$.

因此，在前期 2(5H)-呋喃酮化学研究的基础上 ${ }^{[6,12]}$,

\footnotetext{
*E-mail: wangzy@scnu.edu.cn, chenrh@gdyzy.edu.cn

Received May 18, 2015; revised June 27, 2015; published online July 8, 2015

Project supported by the Natural Science Foundation of Guangdong Province (Nos. 2014A030313429, S2011010001556), the 3rd Talents Special Funds of Guangdong Higher Education (No. Guangdong-Finance-Education [2011]431), the National Natural Science Foundation of China (No. 31200439) and the Natural Science Project of Guangdong Food and Drug Vocational College (No. 2014YZ007).

广东省自然科学基金(Nos. 2014A030313429, S2011010001556)、广东省高等学校人才引进专项资金(No. 粤财教[2011]431 号)、国家自然科学基金(No. 31200439)、广东食品药品职业学院自然科学研究(No. 2014YZ007)资助项目.
} 


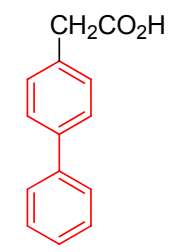

Felbinac

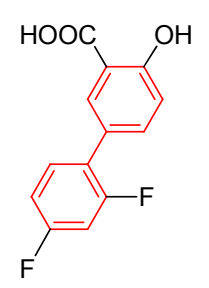

Diflunisal

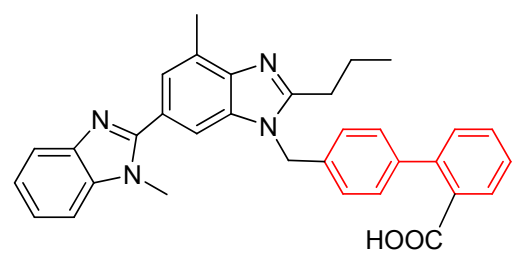

Telmisartan

图 1 一些具有联苯结构单元的生物活性化合物

Figure 1 Some bioactive compounds with biphenyl unit

特别是基于针对 $2(5 H)$-呋喃酮类合成子 5 -位进行修饰改 造而引入联苯结构的研究 ${ }^{[1]}$, 本文继续通过 5-位、4-位 连续进行修饰而设计、合成了系列同时含有 $2(5 H)$-呋喃 酮和联苯两种生物活性单元的新 2(5H)-呋喃酮衍生物 5 (Scheme 1). 系列新化合物的合成(含化合物 3), 一方面 继续为研究较少的 $2(5 H)$-呋喃酮类合成子 5 -位修饰 $[5,13]$ 与不同着基化合物合成芳香醚类 ${ }^{[11,14]}$ 提供了参考, 同时 也为下一步生物活性测试中与前文 ${ }^{[11]}$ 对比研究不同位 点烷基修饰带来的生物活性影响奠定了基础.

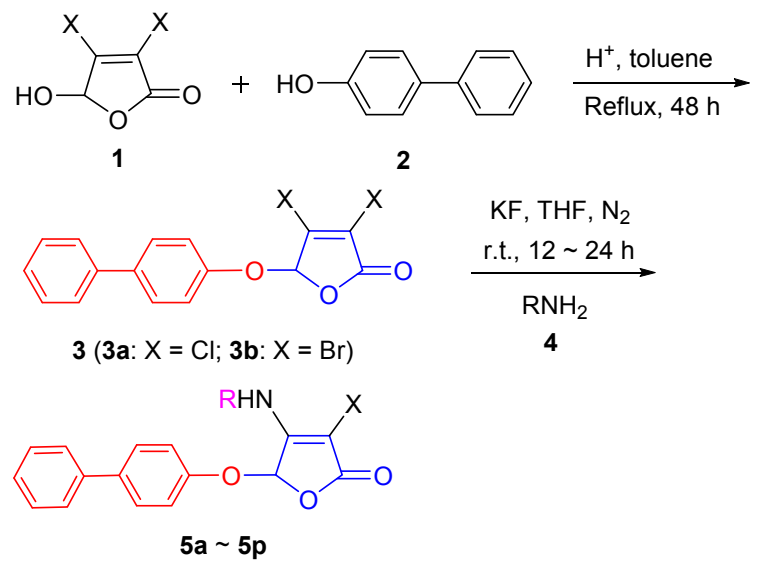

$\mathrm{X}=\mathrm{Cl}, \mathrm{Br}$; $\mathrm{R}=\left(\mathrm{CH}_{2}\right)_{n} \mathrm{CH}_{3}(n=1,6,7,9,11,13), \mathrm{CH}_{2} \mathrm{CH}=\mathrm{CH}_{2}, \mathrm{H}_{2} \mathrm{CH}_{2} \mathrm{C}$

图式 1 目标化合物 5 的合成路线

Scheme 1 The synthetic route of target compounds 5

\section{1 结果与讨论}

\section{1 目标化合物的合成}

化合物 3 是参考文献[11]的反应条件进行合成的 (Scheme 1), 产率 46\% 54\%, 这进一步表明以廉价易
得的 $\mathrm{H}_{2} \mathrm{SO}_{4}$ 作为催化剂应用于酚类化合物与多官能团 差基化合物的直接脱水醚化是可行的. 以化合物 3 为中 间体与不同的代表性脂肪胺 4 进行串联 Michael 加成消除反应，参考文献[15]的反应条件进行(TLC 跟踪，室 温反应 12〜24 h), 以 43\% 79\%的分离产率(绝大部分 等于或高于 $55 \%$, 表 1), 获得了目标产物 3-卤-4-脂肪胺 基-5-联苯氧基-2(5H)-呋喃酮(5a $\sim 5 \mathbf{p}$ ) (Scheme 1).

2(5H)-呋喃酮中间体 $\mathbf{3}$ 与亲核试剂脂肪胺 $\mathbf{4}$ 的反应 是先经过 Michael 加成反应, 再经消除反应, 最终生成 目标产物 $\mathbf{5}^{[15]}$. 由表 1 可知, 以 3,4-二溴-5-联苯氧基$2(5 H)$ 呋喃酩 $(3 \mathbf{b})$ 为中间体而胺类化合物 4 相同时，化合 物 5 的产率通常比中间体为 3,4-二氯-5-联苯氧基-2 $(5 H)$

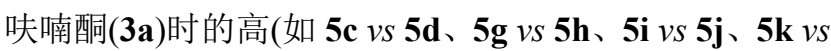
$5 \mathbf{1} 、 5 \mathbf{m} v s \mathbf{n} 、 50$ vs $5 \mathbf{p}$ 等), 其原因可能是消除反应为决 速步; 而在这一步骤中, 溴原子较氯原子更易离去.

此外，不同的脂肪胺 4 与相同的 3,4-二卤-5-联苯氧 基-2(5H) 呋喃酮中间体 $\mathbf{3}$ 反应时，也会对目标化合物 $\mathbf{5}$ 的产率有较大的影响. 一般而言, 长链脂肪胺 $\mathbf{4}$ 的反应 效果普遍比短链脂肪胺的反应效果好, 如 $5 \mathrm{k} \sim 5 \mathrm{p}$ 的产 率都在 63\%以上(表 1, Entries 11 16), 而 5a $\sim 5 \mathbf{j}$ 中大部 分低于 $60 \%$ (表 1, Entries 1 10). 究其原因, 可能是因 为长烷基链具有更好的推电子作用, 有利于脂肪胺 4 作 为一个更有效的亲核试剂进行第一步的 Michael 加成反 应.

另外, 当丙烯胺 4b 与 3,4-二溴-5-联苯氧基-2(5H)呋喃酮 (3b) 反应时, 由于丙烯胺的特殊结构和溴代 2(5H)-呋喃酮中间体共同影响, 使得 $\mathbf{5 d}$ 的收率达到最高 (79\%，表 1, Entry 4).

\section{2 新目标化合物的结构表征}

红外光谱中, 化合物吸收峰在 $3360 \sim 3260 \mathrm{~cm}^{-1}$ 处 为 $\mathrm{N}-\mathrm{H}$ 伸缩振动峰, 在 $3190 \sim 3030 \mathrm{~cm}^{-1}$ 处的吸收是 不饱和 $\mathrm{C}-\mathrm{H}$ 的伸缩振动吸收峰, 在 $2930 \sim 2850 \mathrm{~cm}^{-1}$ 处的吸收是饱和 $\mathrm{C}-\mathrm{H}$ 的伸缩振动吸收峰, 在 1763 $1745 \mathrm{~cm}^{-1}$ 的强吸收峰为 $\mathrm{C}=\mathrm{O}$ 伸缩振动吸收峰, 在 $1660 \sim 1643 \mathrm{~cm}^{-1}$ 强的吸收峰为 $\mathrm{C}=\mathrm{C}$ 伸缩振动吸收峰. 在 $1605 \sim 1450 \mathrm{~cm}^{-1}$ 处吸收峰为苯环骨架伸缩振动峰, 在 $1355 \sim 1368 \mathrm{~cm}^{-1}$ 左右的吸收峰为 $\mathrm{C}-\mathrm{N}$ 伸缩振动振 动峰.

同时, 1305 1004 $\mathrm{cm}^{-1}$ 处的吸收是 $\mathrm{C}-\mathrm{O}-\mathrm{C}$ 伸缩 振动, 975 940 $\mathrm{cm}^{-1}$ 处的吸收是 $\mathrm{C}-\mathrm{O}-\mathrm{C}$ 弯曲振动, $830 \mathrm{~cm}^{-1}$ 左右有苯环对二取代的吸收峰, 768 和 698 $\mathrm{cm}^{-1}$ 左右有苯环单取代的吸收峰. 另外, 在 $740 \mathrm{~cm}^{-1}$ 左 右有 $\mathrm{C}-\mathrm{Cl}$ 的伸缩振动吸收峰, $500 \sim 520 \mathrm{~cm}^{-1}$ 处为 $\mathrm{C}-$ $\mathrm{Br}$ 键的伸缩振动振动峰. 因此, 红外测试结果表明, 目 
表 1 目标化合物 5 的产率 ${ }^{a}$

Table 1 The yields of target compounds 5

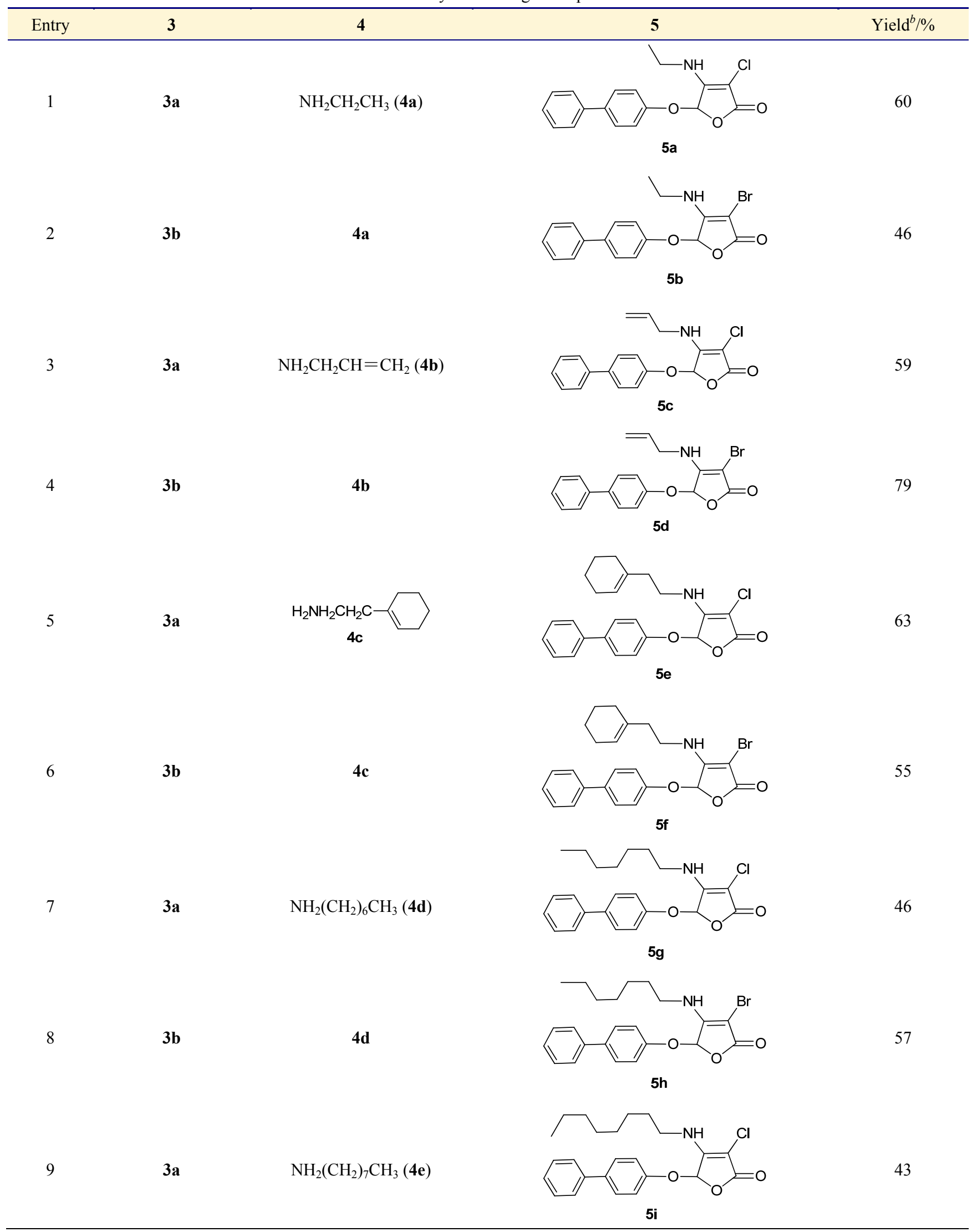




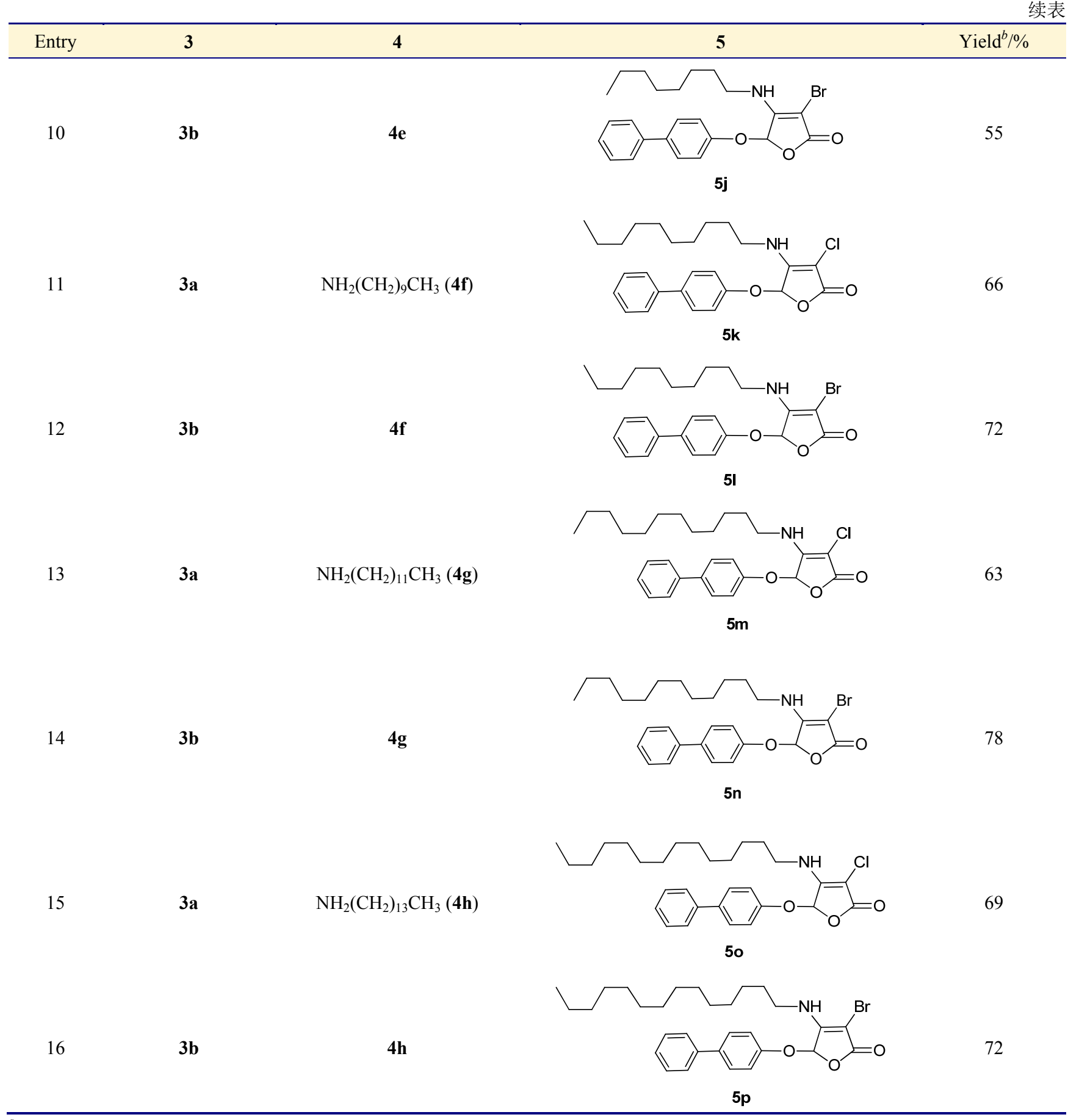

${ }^{a}$ Reaction conditions: aliphatic amine 4 (0.30 mmol), 5-biphenyloxy-3,4-dihalo-2 $(5 H)$-furanone 3 (0.25 mmol), THF (6 mL), KF (0.30 mmol), room temperature, $12 \sim 24 \mathrm{~h}$; ${ }^{b}$ isolated yield.

标化合物 5a $\sim \mathbf{5 p}$ 都含有 $2(5 H)$-呋喃酮环、联苯、 C-X 键和胺基等结构(Scheme 1).

类似地, 264 269 nm 范围的强紫外吸收表明目标 化合物 5 中存在 $\mathrm{C}=\mathrm{C}-\mathrm{C}=\mathrm{O}$ 等共轭结构. 在 ${ }^{1} \mathrm{H}$ NMR 中, $\delta 6.13 \sim 6.25$ 有 $2(5 H)$-呋喃酮环结构的 5-位氢存在; 而在 $\delta 4.96 \sim 5.46$ 处有胺基上 $\mathrm{N}-\mathrm{H}$ 的吸收峰. 结合其 他结构表征, 说明在该反应条件下 $2(5 H)$-呋喃酮环结构 没有受到影响而开环. 不仅如此, 化合物 $\mathbf{5 e} \sim \mathbf{5 f}$ 在 $\delta$
5.17 5.99 处有 $=\mathrm{C}-\mathrm{H}$ 的吸收峰.

另外, ${ }^{13} \mathrm{C} \mathrm{NMR}$ 和元素分析数据和相应的理论值一 致，进一步证明了化合物 5 为目标化合物. 更重要的是， 这些结构表征表明，尽管 3,4-二卤-5-联苯氧基- 2(5H)呋 喃酮(3)上含有多种敏感基团, 如羰基、卤素、双键、杂 环、醚键、芳环、内酯等，但该碱催化的串联 Michael 加成一消除反应仍是可行的，而且使用不饱和胺 $4 b 、 4 c$ 时还可引入新的双键. 


\section{2 结论}

在温和而简单的碱催化条件下, 通过高效的串联 Michael 加成-消除反应, 成功合成了 16 个新的具有联 苯结构的 2(5H)-呋喃酮化合物, 并经较为充分的结构表 征和确证. 这些含有多生物活性基团的目标化合物的制 备, 为其下一步的生物活性测试提供了基础.

\section{3 实验部分}

\section{1 仪器与试剂}

X-5 型显微熔点仪, 未校正; 德国 Bruker Vector 33 FT-IR 光谱仪, 液膜法 $\left(\mathrm{CH}_{2} \mathrm{Cl}_{2}\right)$; 美国 Varian DRX-400 $\mathrm{MHz}$ 核磁共振仪(溶剂 $\mathrm{CDCl}_{3}$, 内标 TMS); Shimazu UV-2550 紫外光谱仪; 美国 THEROMO LCQ DECA XP MAX 质谱仪; 美国 Thermo Flashea TM 112 元素分析仪. 所有试剂均为市售分析纯, 未经进一步纯化. 化合物 $\mathbf{1}$, 以呋喃甲醛等市售试剂为原料, 参考文献 $[6,11,12,15]$ 等进行合成。

\section{2 实验方法}

\subsection{1 中间体 3 的合成}

参考文献[11]方法, 在 $100 \mathrm{~mL}$ 圆底烧瓶中加入 2.5 $\mathrm{mmol}$ 粘卤酸 1 和 $3 \mathrm{mmol}$ 联苯酚 2 , 溶于 $60 \mathrm{~mL}$ 无水甲 苯中, 滴加 $1 \sim 2$ 滴浓硫酸, 恒温磁力搅拌回流 $48 \mathrm{~h}$. 反 应结束后, 减压旋干甲苯, 粗产品分别用饱和碳酸氢 钠、去离子水洗涤, 乙酸乙酯萃取有机层. 有机层用无 水硫酸镁干燥，柱层析分离得到目标化合物 $\mathbf{3 a} \sim \mathbf{3 b}$.

3,4-二氯-5-联苯氧基-2 $(5 H)$ 呋喃酮(3a): 白色固体, 产率 54\%. m.p. $124.5 \sim 126.1{ }^{\circ} \mathrm{C}$; UV-Vis $\left(\mathrm{CH}_{2} \mathrm{Cl}_{2}\right) \lambda_{\max }$ : $249 \mathrm{~nm} ;{ }^{1} \mathrm{H}$ NMR $\left(\mathrm{CDCl}_{3}, 400 \mathrm{MHz}\right) \delta: 6.23(\mathrm{~s}, 1 \mathrm{H}), 7.20$ (d, $J=8.0 \mathrm{~Hz}, 2 \mathrm{H}), 7.33$ (t, $J=8.0 \mathrm{~Hz}, 1 \mathrm{H}), 7.38 \sim 7.45$ $(\mathrm{m}, 2 \mathrm{H}), 7.50 \sim 7.58(\mathrm{~m}, 4 \mathrm{H}) ;{ }^{13} \mathrm{C}$ NMR $\left(\mathrm{CDCl}_{3}, 100\right.$ MHz) $\delta$ : 98.1, 116.9, 123.8, 125.9, 126.4, 127.6, 127.8, 136.9, 139.0, 145.9, 154.1, 161.6; IR (film) v: 3030, 2970, 2850, 1799, 1638, 1607, 1514, 1485, 1265, 1215, 1096, $978,833,762,746,696 \mathrm{~cm}^{-1}$. Anal. calcd for $\mathrm{C}_{16} \mathrm{H}_{10} \mathrm{Cl}_{2} \mathrm{O}_{3}$ : C 59.84, H 3.14; found C 59.78, H 3.12.

3,4-二溴-5-联苯氧基-2 $(5 H)$ 呋喃酮 (3b): 白色固体, 产率 46\%. m.p. 95.6 97.4 ${ }^{\circ} \mathrm{C}$; UV-Vis $\left(\mathrm{CH}_{2} \mathrm{Cl}_{2}\right) \lambda_{\text {max }}: 250$ $\mathrm{nm} ;{ }^{1} \mathrm{H}$ NMR $\left(\mathrm{CDCl}_{3}, 400 \mathrm{MHz}\right) \delta: 6.25(\mathrm{~s}, 1 \mathrm{H}), 7.22(\mathrm{~d}$, $J=8.0 \mathrm{~Hz}, 2 \mathrm{H}), 7.34(\mathrm{t}, J=8.0 \mathrm{~Hz}, 1 \mathrm{H}), 7.40 \sim 7.46(\mathrm{~m}$, $2 \mathrm{H}), 7.52 \sim 7.60(\mathrm{~m}, 4 \mathrm{H}) ;{ }^{13} \mathrm{C} \mathrm{NMR}\left(\mathrm{CDCl}_{3}, 100 \mathrm{MHz}\right) \delta$ : $101.5,117.9,119.2,127.0,127.4,128.6,128.9,137.9$, 140.1, 142.6, 155.3, 163.6; IR (film) v: 3073, 2922, 2851, 1784, 1612, 1589, 1524, 1487, 1269, 1228, 1196, 968, 837, $758,690,496 \mathrm{~cm}^{-1}$. Anal. calcd for $\mathrm{C}_{16} \mathrm{H}_{10} \mathrm{Br}_{2} \mathrm{O}_{3}$ : C 46.86,
H 2.46; found C 46.73, H 2.30.

\section{2 .2 目标化合物 $\mathbf{5}$ 的合成}

$50 \mathrm{~mL}$ 二颈烧瓶中加入 $0.25 \mathrm{mmol}$ 中间体 3,4-二卤5-联苯氧基-2(5H)-呋喃酮(3), 抽真空, $\mathrm{N}_{2}$ 保护下加入 6 $\mathrm{mL}$ 新蒸馏过的 $\mathrm{THF}$ 溶解, 加入 $0.30 \mathrm{mmol}$ 脂肪胺 $\mathbf{4}$, 再 加入 $0.30 \mathrm{mmol}$ 碱 KF, TLC 跟踪，反应时间一般为 12 $24 \mathrm{~h}$ (大部分 $12 \mathrm{~h}$ 即可). 反应完毕，用饱和食盐水洗涤、 二氯甲烷萃取处理, 得到的有机层用蒸馏水洗涤、无水 硫酸镁干燥后, 减压旋干, 柱层析分离, 得到目标化合 物 $5 \mathrm{a} \sim 5 \mathrm{p}$.

3-氯-4-乙胺基-5-联苯氧基-2 $(5 H)$-呋喃酮 $(\mathbf{5 a})$ : 淡黄 色固体, 产率 60\%. m.p. $44.2 \sim 45.8{ }^{\circ} \mathrm{C}$; UV-Vis $\left(\mathrm{CH}_{2} \mathrm{Cl}_{2}\right)$ $\lambda_{\text {max }}: 265 \mathrm{~nm} ;{ }^{1} \mathrm{H}$ NMR $\left(400 \mathrm{MHz}, \mathrm{CDCl}_{3}\right) \delta: 1.35$ (t, $J=$ $8.0 \mathrm{~Hz}, 3 \mathrm{H}), 3.52 \sim 3.67(\mathrm{~m}, 2 \mathrm{H}), 5.35(\mathrm{~b}, 1 \mathrm{H}), 6.23(\mathrm{~s}$, $1 \mathrm{H}), 7.21(\mathrm{~d}, J=8.0 \mathrm{~Hz}, 2 \mathrm{H}), 7.34(\mathrm{t}, J=8.0 \mathrm{~Hz}, 1 \mathrm{H})$, $7.41 \sim 7.46(\mathrm{~m}, 2 \mathrm{H}), 7.53 \sim 7.59(\mathrm{~m}, 4 \mathrm{H}) ;{ }^{13} \mathrm{C} \mathrm{NMR}$ $\left(\mathrm{CDCl}_{3}, 100 \mathrm{MHz}\right) \delta: 13.7,31.9,95.7,101.0,117.2,126.9$, 127.3, 128.6, 128.8, 137.3, 142.3, 147.9, 155.2, 161.7; IR (film) $v$ : 3354, 3186, 3078, 2928, 2857, 1757, 1647, 1605, 1514, 1485, 1358, 1265, 1221, 1184, 1076, 966, 837, 766, $743,698 \mathrm{~cm}^{-1}$; ESI-MS $m / z(\%): 328.17\left([\mathrm{M}-\mathrm{H}]^{-}, 100\right)$. Anal. calcd for $\mathrm{C}_{18} \mathrm{H}_{16} \mathrm{ClNO}_{3}$ : C 65.56, $\mathrm{H} 4.89, \mathrm{~N}$ 4.25; found $\mathrm{C} 65.57, \mathrm{H} 4.96, \mathrm{~N} 4.19$.

3-澳-4-乙胺基-5-联苯氧基-2(5H)-呋喃酮 $(5 \mathbf{b})$ : 淡黄 色固体, 产率 46\%. m.p. $45.8 \sim 47.1{ }^{\circ} \mathrm{C}$; UV-Vis $\left(\mathrm{CH}_{2} \mathrm{Cl}_{2}\right)$ $\lambda_{\text {max }}: 269 \mathrm{~nm} ;{ }^{1} \mathrm{H}$ NMR $\left(400 \mathrm{MHz}, \mathrm{CDCl}_{3}\right) \delta: 1.35$ (t, $J=$ $8.0 \mathrm{~Hz}, 3 \mathrm{H}), 3.42 \sim 3.65(\mathrm{~m}, 2 \mathrm{H}), 5.01(\mathrm{~b}, 1 \mathrm{H}), 6.25(\mathrm{~s}$, $1 \mathrm{H}), 7.21(\mathrm{~d}, J=8.0 \mathrm{~Hz}, 2 \mathrm{H}), 7.34(\mathrm{t}, J=8.0 \mathrm{~Hz}, 1 \mathrm{H})$, $7.40 \sim 7.47(\mathrm{~m}, 2 \mathrm{H}), 7.52 \sim 7.59(\mathrm{~m}, 4 \mathrm{H}),{ }^{13} \mathrm{C}$ NMR $(100$ $\left.\mathrm{MHz}, \mathrm{CDCl}_{3}\right) \delta: 16.0,39.3,79.5,95.9,117.2,126.9,127.3$, 128.6, 128.8, 137.3, 140.2, 155.3, 156.1, 166.9; IR (film) $v: 3320,3078,3036,2922,2851,1757,1643,1602,1514$, 1485, 1356, 1265, 1217, 1190, 1095, 951, 837, 764, 698, $503 \mathrm{~cm}^{-1}$; ESI-MS $m / z$ (\%): $372.15\left([\mathrm{M}-\mathrm{H}]^{-}\right.$, 99). Anal. calcd for $\mathrm{C}_{18} \mathrm{H}_{16} \mathrm{BrNO}_{3}$ : C 57.77, $\mathrm{H}$ 4.31, N 3.74; found $\mathrm{C}$ 57.86, H 4.42, N 3.67.

3-氯-4-烯丙胺基-5-联苯氧基-2 $(5 H)$-呋喃酮 $(\mathbf{5 c})$ : 淡 黄色固体，产率 59\%. m.p. 87.6 88.9 ${ }^{\circ} \mathrm{C}$; UV-Vis $\left(\mathrm{CH}_{2} \mathrm{Cl}_{2}\right) \lambda_{\text {max }}: 265 \mathrm{~nm} ;{ }^{1} \mathrm{H}$ NMR $\left(400 \mathrm{MHz}, \mathrm{CDCl}_{3}\right) \delta$ : $4.10(\mathrm{~s}, 2 \mathrm{H}), 5.21 \sim 5.31(\mathrm{~m}, 2 \mathrm{H}), 5.34(\mathrm{~b}, 1 \mathrm{H}), 5.84 \sim 5.99$ (m, 1H), $6.22(\mathrm{~s}, 1 \mathrm{H}), 7.17$ (d, $J=8.0 \mathrm{~Hz}, 2 \mathrm{H}), 7.33$ (t, $J=$ $8.0 \mathrm{~Hz}, 1 \mathrm{H}), 7.38 \sim 7.46(\mathrm{~m}, 2 \mathrm{H}), 7.50 \sim 7.58(\mathrm{~m}, 4 \mathrm{H}) ;{ }^{13} \mathrm{C}$ NMR $\left(100 \mathrm{MHz}, \mathrm{CDCl}_{3}\right) \delta: 46.2,95.1,100.3,117.1$, $118.0,126.9,127.3,128.5,128.9,133.3,137.2,140.1$, 155.2, 156.3, 166.8; IR (film) v: 3312, 3078, 3036, 2928, 
2857, 1762, 1659, 1601, 1514, 1485, 1358, 1265, 1221, 1194, 1074, 974, 837, 766, 746, $698 \mathrm{~cm}^{-1}$; ESI-MS $\mathrm{m} / \mathrm{z}$ (\%): $340.29\left([\mathrm{M}-\mathrm{H}]^{-}, 100\right)$. Anal. calcd for $\mathrm{C}_{19} \mathrm{H}_{16} \mathrm{ClNO}_{3}$ C 66.77, H 4.72, N 4.10; found C 66.86, H 4.69, N 4.17.

3-澳-4-烯丙胺基-5-联苯氧基-2 $(5 H)$-呋喃酮 (5d): 淡 黄色固体, 产率 79\%. m.p. 92.5 94.3 ${ }^{\circ} \mathrm{C}$; UV-Vis $\left(\mathrm{CH}_{2} \mathrm{Cl}_{2}\right) \lambda_{\text {max }}: 269 \mathrm{~nm} ;{ }^{1} \mathrm{H}$ NMR $\left(400 \mathrm{MHz}, \mathrm{CDCl}_{3}\right) \delta$ : $4.08(\mathrm{~s}, 2 \mathrm{H}), 5.21 \sim 5.29(\mathrm{~m}, 2 \mathrm{H}), 5.46(\mathrm{~b}, 1 \mathrm{H}), 5.85 \sim 5.95$ (m, 1H), $6.23(\mathrm{~s}, 1 \mathrm{H}), 7.17(\mathrm{~d}, J=8.0 \mathrm{~Hz}, 2 \mathrm{H}), 7.32(\mathrm{t}, J=$ $8.0 \mathrm{~Hz}, 1 \mathrm{H}), 7.38 \sim 7.45(\mathrm{~m}, 2 \mathrm{H}), 7.50 \sim 7.56(\mathrm{~m}, 4 \mathrm{H}) ;{ }^{13} \mathrm{C}$ NMR (100 MHz, $\left.\mathrm{CDCl}_{3}\right) \delta: 46.3,84.8,95.9,117.1,118.1$, $126.9,127.3,128.5,128.9,133.3,137.1,140.1,155.2$, 159.0, 167.1; IR (film) v: 3306, 3078, 3036, 2928, 2857, $1757,1647,1518,1450,1358,1265,1223,1190,1074$, 941, 833, 764, 698, $501 \mathrm{~cm}^{-1}$; ESI-MS $\mathrm{m} / z$ (\%): 384.24 $\left([\mathrm{M}-\mathrm{H}]^{-}\right.$, 95). Anal. calcd for $\mathrm{C}_{19} \mathrm{H}_{16} \mathrm{BrNO}_{3} \mathrm{C} 59.08, \mathrm{H}$ 4.18, N 3.63; found C 59.06, H 4.29, N 3.77.

3-氯-4-(1-环己烯基)乙胺基-5-联苯氧基-2(5H)-呋喃 酩(5e): 淡黄色固体, 产率 63\%. m.p. 101.9 103.9 ${ }^{\circ} \mathrm{C}$; UV-Vis $\left(\mathrm{CH}_{2} \mathrm{Cl}_{2}\right) \lambda_{\max }: 265 \mathrm{~nm} ;{ }^{1} \mathrm{H}$ NMR $(400 \mathrm{MHz}$, $\left.\mathrm{CDCl}_{3}\right) \delta: 1.57 \sim 1.66(\mathrm{~m}, 4 \mathrm{H}), 1.94 \sim 2.04(\mathrm{~m}, 4 \mathrm{H}), 2.30$ (t, $J=8.0 \mathrm{~Hz}, 2 \mathrm{H}), 3.44 \sim 3.69(\mathrm{~m}, 2 \mathrm{H}), 5.01(\mathrm{~b}, 1 \mathrm{H}), 5.18$ (t, $J=8.0 \mathrm{~Hz}, 1 \mathrm{H}), 6.19(\mathrm{~s}, 1 \mathrm{H}), 7.19(\mathrm{~d}, J=8.0 \mathrm{~Hz}, 2 \mathrm{H})$, $7.34(\mathrm{t}, J=8.0 \mathrm{~Hz}, 1 \mathrm{H}), 7.40 \sim 7.46(\mathrm{~m}, 2 \mathrm{H}), 7.53 \sim 7.59$ (m, 4H); ${ }^{13} \mathrm{C}$ NMR $\left(100 \mathrm{MHz}, \mathrm{CDCl}_{3}\right) \delta: 22.2,22.7,25.3$, 27.9, 38.4, 41.4, 95.2, 103.1, 117.2, 125.4, 126.9, 127.3, 128.6, 128.8, 133.2, 137.3, 140.2, 152.6, 155.3, 160.6; IR (film) $v: 3264,3078,2928,2857,1757,1647,1535,1518$, 1485, 1368, 1265, 1233, 1188, 1086, 962, 837, 764, 745, $698 \mathrm{~cm}^{-1}$; ESI-MS m/z (\%): $408.45\left([\mathrm{M}-\mathrm{H}]^{-}, 100\right)$. Anal. calcd for $\mathrm{C}_{24} \mathrm{H}_{24} \mathrm{ClNO}_{3}$ : C 70.32, H 5.90, N 3.42; found $\mathrm{C}$ 70.53, H 5.86, N 3.57 .

3-溴-4-(1-环己烯基)乙胺基-5-联苯氧基-2(5H)-呋喃 酮(5f): 淡黄色固体，产率 55\%. m.p. 102.2 103.5 ${ }^{\circ} \mathrm{C}$; UV-Vis $\left(\mathrm{CH}_{2} \mathrm{Cl}_{2}\right) \lambda_{\max }: 267 \mathrm{~nm} ;{ }^{1} \mathrm{H}$ NMR $(400 \mathrm{MHz}$, $\left.\mathrm{CDCl}_{3}\right) \delta: \quad 1.57 \sim 1.66(\mathrm{~m}, 4 \mathrm{H}), 1.90 \sim 1.95(\mathrm{~m}, 2 \mathrm{H})$, $2.01 \sim 2.06(\mathrm{~m}, 2 \mathrm{H}), 2.29(\mathrm{t}, J=8.0 \mathrm{~Hz}, 2 \mathrm{H}), 3.41 \sim 3.66$ $(\mathrm{m}, 2 \mathrm{H}), 5.14(\mathrm{~b}, 1 \mathrm{H}), 5.55 \sim 5.59(\mathrm{~m}, 1 \mathrm{H}), 6.21(\mathrm{~s}, 1 \mathrm{H})$, $7.19(\mathrm{~d}, J=8.0 \mathrm{~Hz}, 2 \mathrm{H}), 7.34(\mathrm{t}, J=8.0 \mathrm{~Hz}, 1 \mathrm{H}), 7.40 \sim$ $7.45(\mathrm{~m}, 2 \mathrm{H}), 7.52 \sim 7.58(\mathrm{~m}, 4 \mathrm{H}) ;{ }^{13} \mathrm{C}$ NMR $(100 \mathrm{MHz}$, $\left.\mathrm{CDCl}_{3}\right) \delta: 22.2,22.7,25.3,27.9,38.3,41.5,96.0,101.0$, $117.2,125.5,126.9,127.3,128.6,128.9,133.2,137.2$, 140.2, 151.4, 155.3, 168.4; IR (film) v: 3312, 3073, 3030, 2928, 2857, 1753, 1647, 1601, 1514, 1485, 1358, 1265, 1221, 1074, 1007, 947, 837, 764, 698, $501 \mathrm{~cm}^{-1}$; ESI-MS $m / z$ (\%): $452.39\left([\mathrm{M}-\mathrm{H}]^{-}\right.$, 100). Anal. calcd for $\mathrm{C}_{24} \mathrm{H}_{24} \mathrm{BrNO}_{3}$ : C 63.44, H 5.32, N 3.08; found C $63.35 \mathrm{H}$ $5.46, \mathrm{~N} 3.17$.

3-氯-4-庚胺基-5-联苯氧基-2 $(5 H)$-呋喃酮 $(\mathbf{5 g})$ ：淡黄 色固体, 产率 $46 \%$. m.p. $56.7 \sim 58.3{ }^{\circ} \mathrm{C}$; UV-Vis $\left(\mathrm{CH}_{2} \mathrm{Cl}_{2}\right)$ $\lambda_{\text {max }}: 265 \mathrm{~nm} ;{ }^{1} \mathrm{H}$ NMR $\left(400 \mathrm{MHz}, \mathrm{CDCl}_{3}\right) \delta: 0.86-0.89(\mathrm{~m}$, $3 \mathrm{H}), 1.28 \sim 1.33(\mathrm{~m}, 8 \mathrm{H}), 1.63 \sim 1.72(\mathrm{~m}, 2 \mathrm{H}), 3.43 \sim 3.56$ (m, 2H), $5.06(\mathrm{~b}, 1 \mathrm{H}), 6.19$ (s, 1H), 7.19 (d, $J=8.0 \mathrm{~Hz}$, 2H), 7.34 (t, $J=8.0 \mathrm{~Hz}, 1 \mathrm{H}), 7.40 \sim 7.45(\mathrm{~m}, 2 \mathrm{H}), 7.53 \sim$ 7.58 (m, 4H); ${ }^{13} \mathrm{C}$ NMR (100 MHz, $\left.\mathrm{CDCl}_{3}\right) \delta: 14.0,22.6$, 26.5, 28.9, 30.7, 31.7, 44.3, 87.7, 95.1, 117.1, 126.9, 127.3, 128.5, 128.8, 137.2, 140.2, 149.6, 155.3, 166.3; IR (film) $v: 3323,3073,2922,2856,1757,1649,1602,1514,1485$, 1362, 1265, 1221, 1194, 1074, 962, 837, 766, 746, 698 $\mathrm{cm}^{-1}$; ESI-MS $\mathrm{m} / \mathrm{z}(\%): 398.45\left([\mathrm{M}-\mathrm{H}]^{-}, 100\right)$. Anal. calcd for $\mathrm{C}_{23} \mathrm{H}_{26} \mathrm{ClNO}_{3}$ : C 69.08, H 6.55, N 3.50; found C 69.01, H 6.68, N 3.59.

3-溴-4-庚胺基-5-联苯氧基-2 $(5 H)$-呋喃酩 $(\mathbf{5 h})$ : 黄色 粘稠液, 产率 57\%. UV-Vis $\left(\mathrm{CH}_{2} \mathrm{Cl}_{2}\right) \lambda_{\text {max }}$ : $268 \mathrm{~nm} ;{ }^{1} \mathrm{H}$ NMR (400 MHz, $\left.\mathrm{CDCl}_{3}\right) \delta: 0.86 \sim 0.89(\mathrm{~m}, 3 \mathrm{H}), 1.27 \sim$ $1.35(\mathrm{~m}, 8 \mathrm{H}), 1.63 \sim 1.70(\mathrm{~m}, 2 \mathrm{H}), 3.33 \sim 3.58(\mathrm{~m}, 2 \mathrm{H})$, $5.14(\mathrm{~b}, 1 \mathrm{H}), 6.22(\mathrm{~s}, 1 \mathrm{H}), 7.19(\mathrm{~d}, J=8.0 \mathrm{~Hz}, 2 \mathrm{H}), 7.33(\mathrm{t}$, $J=8.0 \mathrm{~Hz}, 1 \mathrm{H}), 7.39 \sim 7.49(\mathrm{~m}, 2 \mathrm{H}), 7.50 \sim 7.58(\mathrm{~m}, 4 \mathrm{H})$; ${ }^{13} \mathrm{C}$ NMR $\left(100 \mathrm{MHz}, \mathrm{CDCl}_{3}\right) \delta: 14.0,22.6,26.5,28.9$, $30.6,31.7,44.4,83.6,95.9,117.1,126.9,127.3,128.5$, 128.8, 137.2, 140.2, 155.3, 156.1, 161.8; IR (film) $v: 3300$, 3078, 3036, 2928, 2857, 1753, 1643, 1602, 1518, 1491, 1358, 1265, 1221, 1190, 1074, 951, 833, 764, 698, 501 $\mathrm{cm}^{-1}$; ESI-MS m/z (\%): 442.35 ([M-H] $]^{-}$, 97). Anal. calcd for $\mathrm{C}_{23} \mathrm{H}_{26} \mathrm{BrNO}_{3}$ : C 62.17, H 5.90, N 3.15; found C 62.31, H 5.88, N 3.29.

3-氯-4-辛胺基-5-联苯氧基-2(5H)-呋喃酮(5i)：淡黄 色固体, 产率 43\%. m.p. $47.6 \sim 49.0{ }^{\circ} \mathrm{C}$; UV-Vis $\left(\mathrm{CH}_{2} \mathrm{Cl}_{2}\right)$ $\lambda_{\max }: 266 \mathrm{~nm} ;{ }^{1} \mathrm{H}$ NMR $\left(400 \mathrm{MHz}, \mathrm{CDCl}_{3}\right) \delta: 0.86 \sim 0.89$ $(\mathrm{m}, 3 \mathrm{H}), 1.28 \sim 1.34(\mathrm{~m}, 10 \mathrm{H}), 1.60 \sim 1.67(\mathrm{~m}, 2 \mathrm{H}), 3.40 \sim$ $3.59(\mathrm{~m}, 2 \mathrm{H}), 4.96(\mathrm{~b}, 1 \mathrm{H}), 6.21(\mathrm{~s}, 1 \mathrm{H}), 7.20$ (d, $J=8.0$ $\mathrm{Hz}, 2 \mathrm{H}), 7.34(\mathrm{t}, J=8.0 \mathrm{~Hz}, 1 \mathrm{H}), 7.40 \sim 7.46(\mathrm{~m}, 2 \mathrm{H})$, $7.53 \sim 7.59(\mathrm{~m}, 4 \mathrm{H}) ;{ }^{13} \mathrm{C}$ NMR $\left(100 \mathrm{MHz}, \mathrm{CDCl}_{3}\right) \delta: 13.0$, 21.6, 25.5, 28.1, 28.2, 29.7, 30.7, 43.3, 87.4, 94.1, 116.1, $125.9,126.3,127.5,127.8,136.2,139.1,154.2,156.3$, 166.7; IR (film) v: 3300, 3073, 3030, 2922, 2856, 1757, 1647, 1602, 1518, 1487, 1364, 1265, 1221, 1194, 1076, 962, 833, 768, 743, $694 \mathrm{~cm}^{-1}$; ESI-MS $m / z$ (\%): 412.35 $\left([\mathrm{M}-\mathrm{H}]^{-}, 100\right)$. Anal. calcd for $\mathrm{C}_{24} \mathrm{H}_{28} \mathrm{ClNO}_{3}: \mathrm{C} 69.64, \mathrm{H}$ 6.82, N 3.38; found C 69.57, H 6.97, N 3.49. 
3-溴-4-辛胺基-5-联苯氧基-2(5H)-呋喃酮(5j): 淡黄 色固体, 产率 55\%. m.p. 48.3 49.8 ${ }^{\circ} \mathrm{C}$; UV-Vis $\left(\mathrm{CH}_{2} \mathrm{Cl}_{2}\right)$ $\lambda_{\text {max }}: 266 \mathrm{~nm} ;{ }^{1} \mathrm{H}$ NMR $\left(400 \mathrm{MHz}, \mathrm{CDCl}_{3}\right) \delta: 0.86 \sim 0.89$ $(\mathrm{m}, 3 \mathrm{H}), 1.27 \sim 1.37(\mathrm{~m}, 10 \mathrm{H}), 1.63 \sim 1.68(\mathrm{~m}, 2 \mathrm{H}), 3.34 \sim$ $3.58(\mathrm{~m}, 2 \mathrm{H}), 5.10(\mathrm{~b}, 1 \mathrm{H}), 6.22(\mathrm{~s}, 1 \mathrm{H}), 7.19(\mathrm{~d}, J=8.0$ $\mathrm{Hz}, 2 \mathrm{H}), 7.34(\mathrm{t}, J=8.0 \mathrm{~Hz}, 1 \mathrm{H}), 7.39 \sim 7.46(\mathrm{~m}, 2 \mathrm{H})$, $7.52 \sim 7.58(\mathrm{~m}, 4 \mathrm{H}) ;{ }^{13} \mathrm{C} \mathrm{NMR}\left(100 \mathrm{MHz}, \mathrm{CDCl}_{3}\right) \delta: 13.0$, 21.6, 25.5, 28.1, 28.2, 29.6, 30.7, 43.5, 83.9, 94.9, 116.1, $125.9,126.3,127.5,127.8,136.2,139.1,154.2,157.4$, 166.7; IR (film) v: 3300, 3078, 3036, 2928, 2857, 1753, $1643,1602,1518,1491,1358,1265,1221,1190,1074$, 951, 833, 764, 698, $546 \mathrm{~cm}^{-1}$; ESI-MS m/z (\%): 456.33 $\left([\mathrm{M}-\mathrm{H}]^{-}, 100\right)$. Anal. calcd for $\mathrm{C}_{24} \mathrm{H}_{28} \mathrm{BrNO}_{3}$ : C 62.88, H 6.16, N 3.06; found C 62.97, H 6.18, N 3.01.

3-氯-4-癸胺基-5-联苯氧基-2(5H)-呋喃酮(5k): 淡黄 色固体，产率 66\%. m.p. 50.1 51.7 ${ }^{\circ} \mathrm{C}$; UV-Vis $\left(\mathrm{CH}_{2} \mathrm{Cl}_{2}\right)$ $\lambda_{\text {max }}: 264 \mathrm{~nm} ;{ }^{1} \mathrm{H}$ NMR $\left(400 \mathrm{MHz}, \mathrm{CDCl}_{3}\right) \delta: 0.87(\mathrm{t}, J=$ $8.0 \mathrm{~Hz}, 3 \mathrm{H}), 1.22 \sim 1.30(\mathrm{~m}, 14 \mathrm{H}), 1.59 \sim 1.66(\mathrm{~m}, 2 \mathrm{H})$, $3.36 \sim 3.55(\mathrm{~m}, 2 \mathrm{H}), 5.35(\mathrm{~b}, 1 \mathrm{H}), 6.14(\mathrm{~s}, 1 \mathrm{H}), 7.15(\mathrm{~d}$, $J=8.0 \mathrm{~Hz}, 2 \mathrm{H}), 7.30 \sim 7.35(\mathrm{~m}, 1 \mathrm{H}), 7.38 \sim 7.43(\mathrm{~m}, 2 \mathrm{H})$, $7.50 \sim 7.54(\mathrm{~m}, 4 \mathrm{H}) ;{ }^{13} \mathrm{C} \mathrm{NMR}\left(100 \mathrm{MHz}, \mathrm{CDCl}_{3}\right) \delta: 14.1$, $22.7,26.5,29.2,29.3,29.5,29.6,30.7,31.9,44.2,87.5$, $95.2,117.2,126.9,127.3,128.5,128.9,137.1,140.1$, 155.3, 155.8, 167.8; IR (film) v: 3312, 3078, 3030, 2928, 2857, 1748, 1643, 1602, 1518, 1485, 1364, 1265, 1233, 1109, 1082, 966, 837, 766, 746, $694 \mathrm{~cm}^{-1}$; ESI-MS m/z (\%): $440.47\left([\mathrm{M}-\mathrm{H}]^{-}, 100\right)$. Anal. calcd for $\mathrm{C}_{26} \mathrm{H}_{32}-$ $\mathrm{ClNO}_{3}$ : C 70.65, H 7.30, N 3.14; found C 70.83, H 7.44, N 3.09 .

3-溴-4-癸胺基-5-联苯氧基-2(5H)-呋喃酮(5l): 淡黄 色固体, 产率 72\%. m.p. 49.7 50.7 ${ }^{\circ} \mathrm{C}$; UV-Vis $\left(\mathrm{CH}_{2} \mathrm{Cl}_{2}\right)$ $\lambda_{\text {max }}: 265 \mathrm{~nm} ;{ }^{1} \mathrm{H}$ NMR $\left(400 \mathrm{MHz}, \mathrm{CDCl}_{3}\right) \delta: 0.87(\mathrm{t}, J=$ $8.0 \mathrm{~Hz}, 3 \mathrm{H}), 1.22 \sim 1.32(\mathrm{~m}, 14 \mathrm{H}), 1.59 \sim 1.70(\mathrm{~m}, 2 \mathrm{H})$, $3.25 \sim 3.62(\mathrm{~m}, 2 \mathrm{H}), 5.31(\mathrm{~b}, 1 \mathrm{H}), 6.18(\mathrm{~s}, 1 \mathrm{H}), 7.16(\mathrm{~d}$, $J=8.0 \mathrm{~Hz}, 2 \mathrm{H}), 7.31 \sim 7.36(\mathrm{~m}, 1 \mathrm{H}), 7.38 \sim 7.43(\mathrm{~m}, 2 \mathrm{H})$, $7.50 \sim 7.56(\mathrm{~m}, 4 \mathrm{H}) ;{ }^{13} \mathrm{C} \mathrm{NMR}\left(100 \mathrm{MHz}, \mathrm{CDCl}_{3}\right) \delta: 14.1$, $22.7,26.5,29.2,29.3,29.5,29.6,30.6,31.9,44.3,92.1$, $96.0,117.1,126.9,127.3,128.5,128.9,137.1,140.1$, 155.3, 155.8, 167.6; IR (film) v: 3318, 3073, 3030, 2928, 2857, 1753, 1647, 1602, 1518, 1485, 1364, 1265, 1217, 1101, 1080, 951, 833, 764, 694, $501 \mathrm{~cm}^{-1}$; ESI-MS m/z $(\%): 484.45\left([\mathrm{M}-\mathrm{H}]^{-}, 100\right)$. Anal. calcd for $\mathrm{C}_{26} \mathrm{H}_{32} \mathrm{BrNO}_{3}$ : C 64.20, $\mathrm{H}$ 6.63, N 2.88; found $\mathrm{C} 64.32, \mathrm{H}$ $6.77, \mathrm{~N} 2.81$.

3-氯-4-十二胺基-5-联苯氧基-2(5H)-呋喃酮 (5m):
淡黄色固体，产率 $63 \%$. m.p. 57.6 58.8 ${ }^{\circ} \mathrm{C}$; UV-Vis $\left(\mathrm{CH}_{2} \mathrm{Cl}_{2}\right) \lambda_{\max }: 266 \mathrm{~nm} ;{ }^{1} \mathrm{H} \mathrm{NMR}\left(400 \mathrm{MHz}, \mathrm{CDCl}_{3}\right) \delta$ : $0.85 \sim 0.89(\mathrm{~m}, 3 \mathrm{H}), 1.24 \sim 1.28(\mathrm{~m}, 18 \mathrm{H}), 1.63 \sim 1.68(\mathrm{~m}$, $2 \mathrm{H}), 3.32 \sim 3.61(\mathrm{~m}, 2 \mathrm{H}), 5.20(\mathrm{~b}, 1 \mathrm{H}), 6.16(\mathrm{~s}, 1 \mathrm{H}), 7.17$ $(\mathrm{d}, J=8.0 \mathrm{~Hz}, 2 \mathrm{H}), 7.33$ (t, $J=8.0 \mathrm{~Hz}, 1 \mathrm{H}), 7.39 \sim 7.45$ $(\mathrm{m}, 2 \mathrm{H}), 7.51 \sim 7.56(\mathrm{~m}, 4 \mathrm{H}) ;{ }^{13} \mathrm{C} \mathrm{NMR}\left(100 \mathrm{MHz}, \mathrm{CDCl}_{3}\right)$ $\delta: 14.1,22.7,26.5,29.2,29.4,29.5,29.6,29.7,29.8,30.7$, $31.9,44.2$, 90.6, 95.1, 117.2, 126.9, 127.3, 128.5, 128.8, 137.1, 140.1, 155.3, 156.1, 167.8; IR (film) v: 3281, 3084, 2922, 2851, 1746, 1647, 1550, 1518, 1485, 1364, 1304, 1236, 1113, 1080, 972, 837, 766, 746, $694 \mathrm{~cm}^{-1}$; ESI-MS $m / z \quad(\%): 468.41\left([\mathrm{M}-\mathrm{H}]^{-}, 100\right)$. Anal. calcd for $\mathrm{C}_{28} \mathrm{H}_{36} \mathrm{ClNO}_{3}$ : C 71.55, $\mathrm{H} 7.72, \mathrm{~N} 2.98$; found $\mathrm{C} 71.63, \mathrm{H}$ 7.64, N 3.01 .

3-溴-4-十二胺基-5-联苯氧基-2(5H)-呋喃酮(5n): 淡 黄色固体，产率 78\%. m.p. 49.6 $50.4{ }^{\circ} \mathrm{C}$; UV-Vis $\left(\mathrm{CH}_{2} \mathrm{Cl}_{2}\right) \lambda_{\max }: 268 \mathrm{~nm} ;{ }^{1} \mathrm{H} \mathrm{NMR}\left(400 \mathrm{MHz}, \mathrm{CDCl}_{3}\right) \delta$ : $0.85 \sim 0.90(\mathrm{~m}, 3 \mathrm{H}), 1.22 \sim 1.30(\mathrm{~m}, 18 \mathrm{H}), 1.62 \sim 1.70(\mathrm{~m}$, 2H), $3.25 \sim 3.64(\mathrm{~m}, 2 \mathrm{H}), 5.29(\mathrm{~b}, 1 \mathrm{H}), 6.18(\mathrm{~s}, 1 \mathrm{H}), 7.17$ $(\mathrm{d}, J=8.0 \mathrm{~Hz}, 2 \mathrm{H}), 7.32(\mathrm{t}, J=8.0 \mathrm{~Hz}, 1 \mathrm{H}), 7.38 \sim 7.45$ (m, 2H), $7.50 \sim 7.56(\mathrm{~m}, 4 \mathrm{H}) ;{ }^{13} \mathrm{C}$ NMR (100 MHz, $\left.\mathrm{CDCl}_{3}\right)$ $\delta: 14.1,22.7,26.5,29.2,29.4,29.5,29.6,29.7,29.8,30.6$, $31.9,44.3,88.7,95.9,117.1,126.9,127.3,128.5,128.8$, 137.1, 140.1, 156.3, 159.2, 167.1; IR (film) v: 3294, 3078, 3030, 2922, 2851, 1757, 1643, 1602, 1518, 1487, 1364, 1265, 1221, 1188, 1076, 951, 833, 764, 698, $507 \mathrm{~cm}^{-1}$; ESI-MS $m / z(\%): 512.47\left([\mathrm{M}-\mathrm{H}]^{-}, 100\right)$. Anal. calcd for $\mathrm{C}_{28} \mathrm{H}_{36} \mathrm{BrNO}_{3}$ : C 65.37, $\mathrm{H}$ 7.05, N 2.72; found $\mathrm{C} 65.42, \mathrm{H}$ $7.17, \mathrm{~N} 2.61$.

3-氯-4-十四胺基-5-联苯氧基-2(5H)-呋喃酮(5o): 淡 黄色固体，产率 69\%. m.p. 69.7 71.5 ${ }^{\circ} \mathrm{C}$; UV-Vis $\left(\mathrm{CH}_{2} \mathrm{Cl}_{2}\right) \lambda_{\max }: 266 \mathrm{~nm} ;{ }^{1} \mathrm{H}$ NMR $\left(400 \mathrm{MHz}, \mathrm{CDCl}_{3}\right) \delta$ : $0.85 \sim 0.90(\mathrm{~m}, 3 \mathrm{H}), 1.22 \sim 1.30(\mathrm{~m}, 18 \mathrm{H}), 1.60 \sim 1.68(\mathrm{~m}$, $2 \mathrm{H}), 3.33 \sim 3.56(\mathrm{~m}, 2 \mathrm{H}), 5.35(\mathrm{~b}, 1 \mathrm{H}), 6.13(\mathrm{~s}, 1 \mathrm{H}), 7.15$ $(\mathrm{d}, J=8.0 \mathrm{~Hz}, 2 \mathrm{H}), 7.32$ (t, $J=8.0 \mathrm{~Hz}, 1 \mathrm{H}), 7.38 \sim 7.44$ $(\mathrm{m}, 2 \mathrm{H}), 7.50 \sim 7.55(\mathrm{~m}, 4 \mathrm{H}) ;{ }^{13} \mathrm{C}$ NMR $\left(100 \mathrm{MHz}, \mathrm{CDCl}_{3}\right)$ $\delta: 14.1,22.7,26.5,29.2,29.3,29.4,29.5,29.6,29.7,29.8$, $29.9,30.7,31.9,44.2,88.1,95.2,117.2,126.9,127.3$, 128.4, 128.9, 137.1, 140.1, 153.0, 155.3, 167.3; IR (film) $v: 3300,3078,3036,2928,2857,1746,1639,1553,1514$, 1485, 1362, 1223, 1188, 1107, 1080, 966, 833, 760, 741, $698 \mathrm{~cm}^{-1}$; ESI-MS m/z (\%): $496.49\left([\mathrm{M}-\mathrm{H}]^{-}, 100\right)$. Anal. calcd for $\mathrm{C}_{30} \mathrm{H}_{40} \mathrm{ClNO}_{3}$ : C 72.34, $\mathrm{H}$ 8.09, N 2.81; found $\mathrm{C} 72.51, \mathrm{H} 8.18, \mathrm{~N} 2.61$.

3-溴-4-十四胺基-5-联苯氧基-2(5H)-呋喃酮(5p)：淡 
黄色固体，产率 $72 \%$. m.p. $72.3 \sim 73.7{ }^{\circ} \mathrm{C}$; UV-Vis $\left(\mathrm{CH}_{2} \mathrm{Cl}_{2}\right) \lambda_{\text {max }}: 268 \mathrm{~nm} ;{ }^{1} \mathrm{H}$ NMR $\left(400 \mathrm{MHz}, \mathrm{CDCl}_{3}\right) \delta$ : $0.85 \sim 0.90(\mathrm{~m}, 3 \mathrm{H}), 1.23 \sim 1.29(\mathrm{~m}, 18 \mathrm{H}), 1.59 \sim 1.67(\mathrm{~m}$, $2 \mathrm{H}), 3.29 \sim 3.56(\mathrm{~m}, 2 \mathrm{H}), 5.37(\mathrm{~b}, 1 \mathrm{H}), 6.16(\mathrm{~s}, 1 \mathrm{H}), 7.15$ $(\mathrm{d}, J=8.0 \mathrm{~Hz}, 2 \mathrm{H}), 7.31(\mathrm{t}, J=8.0 \mathrm{~Hz}, 1 \mathrm{H}), 7.38 \sim 7.43$ (m, $2 \mathrm{H}), 7.50 \sim 7.55(\mathrm{~m}, 4 \mathrm{H}) ;{ }^{13} \mathrm{C} \mathrm{NMR}\left(100 \mathrm{MHz}, \mathrm{CDCl}_{3}\right)$ $\delta: 14.2,22.7,26.5,29.3,29.4,29.5,29.6,29.7,29.8,29.9$, $30.6,32.0,44.3,96.0,100.0,117.1,126.9,127.3,128.5$, 128.9, 137.0, 140.1, 155.3, 157.0, 167.4; IR (film) v: 3294, 3084, 3030, 2928, 2862, 1753, 1638, 1551, 1518, 1487, 1364, 1263, 1221, 1098, 1076, 951, 833, 764, 694, 501 $\mathrm{cm}^{-1}$; ESI-MS $m / z$ (\%): $540.41\left([\mathrm{M}-\mathrm{H}]^{-}, 100\right)$. Anal. calcd for $\mathrm{C}_{30} \mathrm{H}_{40} \mathrm{BrNO}_{3}: \mathrm{C} 66.41, \mathrm{H}$ 7.43, N 2.58; found C 66.51, H 7.42, N 2.69.

辅助材料(Supporting Information) 所有 18 个新合成 化合物 3a $\sim 3 b 、 5 a \sim 5 p$ 的核磁共振氢谱、核磁共振碳 谱和质谱图. 这些材料可以免费从本刊网站(http://siocjournal.cn/)上下载.

\section{References}

[1] (a) Trost, B. M.; Burns, A. C.; Bartlett, M. J.; Tautz, T.; Weiss, A. H. J. Am. Chem. Soc. 2012, 134, 1474.

(b) Xie, Y.-Z.; Wang, N.; Cheng, B.; Zhai, H.-B. Org. Lett. 2012, 14,3 .

(c) Tatton, M. R.; Simpson, I.; Donohoe, T. J. Chem. Commun. 2014, 50, 11314.

(d) Müller, D. S.; Untiedt, N. L.; Dieskau, A. P.; Lackner, G. L.; Overman, L. E. J. Am. Chem. Soc. 2015, 137, 660.

[2] Guerrero, M. D.; Aquino, M.; Bruno, I.; Terencio, M. C.; Paya, M.; Riccio, R.; Gomez-Paloma, L. J. Med. Chem. 2007, 50, 2176.

[3] Wei, M.-X.; Feng, L.; Li, X.-Q.; Zhou, X.-Z.; Shao, Z.-H. Eur. J. Med. Chem. 2009, 44, 3340.

[4] (a) Kamal, A.; Suresh, P.; Mallareddy, A.; Kumar, B. A.; Reddy, P. V.; Raju, P.; Tamboli, J. R.; Shaik, T. B.; Jain, N.; Kalivendi, S. V. Bioorg. Med. Chem. 2011, 19, 2349.

(b) Bromhead, L. J.; Visser, J.; McErlean, C. S. P. J. Org. Chem. 2013, 79, 1516.

(c) Pereira, U. A.; Barbosa, L. C. A.; Maltha, C. R. A. Bioorg. Med. Chem. Lett. 2014, 24, 1052.

[5] Sarma, K. D.; Zhang, J.; Curran, T. T. J. Org. Chem. 2007, 72, 3311.

[6] (a) Tan, Y.-H.; Li, J.-X.; Xue, F.-L.; Qi, J.; Wang, Z.-Y. Tetrahedron 2012, 68, 2827.

(b) Shi, J.; Tang, X.-D.; Wu, Y.-C.; Li, H.-N.; Song, L.-J.; Wang, Z.-Y. Eur. J. Org. Chem. 2015, 1193.

[7] Janody, S.; Hermange, P.; Retailleau, P.; Thierry, J.; Dodd, R. H. J. Org. Chem. 2014, 79, 5673.

[8] (a) Latypova, L. Z.; Saigitbatalova, E. S.; Chulakova, D. R.; Lodochnikova, O. A.; Kurbangalieva, A. R.; Berdnikov, E. A.; Chmutova, G. A. Russ. J. Org. Chem. 2014, 50, 521.

(b) Liu, J.; Li, Z.-C.; Tong, P.; Xie, Z.-X.; Zhang, Y.; Li, Y. J. Org. Chem. 2015, 80, 1632.

(c) Wang, X.-D.; Wei, W.; Deng, R. C; Zhou, S.-S.; Zhang, L.; Lin,
X.-Y.; Xiao, Z.-P. Chin. J. Org. Chem. 2014, 34, 1773 (in Chinese). (王旭东, 魏伟, 邓瑞成, 周沙沙, 张蕾, 林肖依, 肖竹平, 有机 化学, 2014, 34, 1773.)

(d) Wei, M.-X.; Gao, X.-H.; Zhang, H.; Li, X.-Q. Chin. J. Org. Chem. 2015, 35, 439 (in Chinese).

(魏梦雪, 高晓慧, 张和, 李学强, 有机化学, 2015, 35, 439.)

[9] (a) Hajduk, P. J.; Sheppard, G.; Nettesheim, D. G.; Hajduk, P. J.; Sheppard, G.; Nettesheim, D. G.; Olejniczak, E. T.; Shuker, S. B.; Meadows, R. P.; Steinman, D. H.; Carrera, G. M.; Marcotte, P. A.; Severin, J.; Walter, K.; Smith, H.; Gubbins, E.; Simmer, R.; Holzman, T. F.; Morgan, D. W.; Davidsen, S. K.; Summers, J. B.; Fesik, S. W. J. Am. Chem. Soc. 1997, 119, 5818.

(b) Kim, S. H.; Kim, J. G. Bull. Korean Chem. Soc. 2011, 32, 341.

(c) Zhou, C.; Li, Y.-M.; Lu, Y.; Zhang, R.; Jin, K.; Fu, X.-M.; Duan, C.-Y. Chin. J. Chem. 2013, 31, 1269.

(d) Hawksworth, E. L.; Andrews, P. C.; Lie, W.; Lai, B.; Dillon, C. T. J. Inorg. Biochem. 2014, 135, 28.

(e) Belema, M.; Nguyen, V. N.; Bachand, C.; Deon, D. H.; Goodrich, J. T.; James, C. A.; Lavoie, R.; Lopez, O. D.; Martel, A.; Romine, J. L.; Ruediger, E. H.; Snyder, L. B.; St Laurent, D. R.; Yang, F.-K.; Zhu, J.-L.; Wong, H.-S.; Langley, D. R.; Adams, S. P.; Cantor, G. H.; Chimalakonda, A.; Fura, A.; Johnson, B. M.; Knipe, J. O.; Parker, D. D.; Santone, K. S.; Fridell, R. A.; Lemm, J. A.; O'Boyle, D. R.; Colonno, R. J.; Gao, M.; Meanwell, N. A.; Hamann, L. G. J Med Chem. 2014, 57, 2013.

(f) Martin, A. D.; Siamaki, A. R.; Belecki, K.; Gupton, B. F. J. Org. Chem. 2015, 80, 1915.

[10] (a) Ortgies, D. H.; Forgione, P. Synlett 2013, 1715.

(b) Ortgies, D. H.; Barthelme, A.; Aly, S.; Desharnais, B.; Rioux, S.; Forgione, P. Synthesis 2013, 45, 694.

(c) Costa, N. E.; Pelotte, A. L.; Simard, J. M. J. Chem. Educ. 2012, 89, 1064.

(d) Wang, L.-M.; Cheng, S.-X.; Chen, T.; Chang, J.-B. Acta Chim. Sinica 2012, 70, 1201 (in Chinese).

(王利敏, 程森祥, 陈肜, 常俊标, 化学学报, 2012, 70, 1201.)

(e) Jiang, G.-F.; Niu, P.-P.; Zhang, Z.-C.; Yan, B.-M.; Guo, C.-C. J. Hunan Univ. (Nat. Sci. Ed.) 2014, 41, 112 (in Chinese).

(江国防, 牛平平, 张志超, 焉保明, 郭灿城, 湖南大学学报(自 然科学版), 2014, 41, 112.)

(f) Bernaskova, M.; Kretschmer, N.; Schuehly, W.; Huefner, A.; Weis, R.; Bauer, R. Molecules 2014, 19, 1223.

[11] Guan, L.-T.; Mo, G.-Z.; Wu, Y.-C.; Liang, X.-Y.; Luo, Q.-F.; Wang, Z.-Y. Chin. J. Org. Chem. 2015, 35, 1081 (in Chinese).

(关丽涛, 莫广珍, 吴彦城, 梁欣榆, 罗俏芳, 汪朝阳, 有机化学, 2015, 35, 1081.)

[12] (a) Huo, J.-P.; Luo, J.-C.; Wu, W.; Xiong, J.-F.; Mo, G.-Z.; Wang, Z.-Y. Ind. Eng. Chem. Res. 2013, 52, 11850.

(b) Huo, J.-P.; Deng, G.-H.; Wu, W.; Xiong, J.-F.; Zhong, M.-L.; Wang, Z.-Y. Macromol. Rapid Commun. 2013, 34, 1779.

(c) Xue, F.-L.; Peng, P.; Shi, J.; Zhong, M.-L.; Wang, Z.-Y. Synth. Commun. 2014, 44, 1944.

[13] (a) Polezhaeva, N. A.; Kalinina, I. V.; Volodina, Y. M.; Berdnikov, E. A.; Galkin, V. I.; Cherkasov, R. A. Russ. J. Gen. Chem. 2004, 40, 438.

(b) Zhang, J.; Blazecka, P. G.; Curran, T. T. Tetrahedron Lett. 2007, 48, 2611.

(c) Liu, G.-Y.; Guo, B.-Q.; Chen, W.-N.; Cheng, C.; Zhang, Q.-L.; Dai, M.-B.; Sun, J.-R.; Sun, P.-H.; Chen, W.-M. Chem. Biol. Drug Des. 2012, 79, 628.

(d) Claraz, A.; Oudeyer, S.; Levacher, V. Adv. Synth. Catal. 2013, 355,841 .

[14] (a) Murai, M.; Origuchi, K.; Takai, K. Org. Lett. 2014, 16, 3828. 
(b) Veenboer, R. M. P.; Nolan, S. P. Green Chem. 2015, 17, 3819

[15] (a) Chen, R.-H.; Fu, J.-H.; Li, G.-L.; Tan, Y.-H.; Wang, Z.-Y.; Yuan, P. Chin. J. Org. Chem. 2012, 32, 95 (in Chinese).

(陈任宏, 傅建花, 李国良, 谭越河, 汪朝阳, 袁萍, 有机化学, 2012, 32, 95.)

(b) Fu, J.-H.; Wang, Z.-Y.; Huo, J.-P.; Tan, Y.-H.; Zeng, R.-H. Chin. J. Org. Chem. 2012, 32, 104 (in Chinese).

(傅建花, 汪朝阳, 霍景沛, 谭越河, 曾荣华, 有机化学, 2012, 32,104 .) (c) Xue, F.-L.; Li, J.-X.; Wang, Z.-Y.; Xiong, J.-F.; Li, D. Res. Chem. Intermed. 2013, 39, 1153.

(d) Xue, F.-L.; Qi, J.; Peng, P.; Mo, G.-Z.; Wang, Z.-Y. Lett. Org. Chem. 2014, 11, 64.

(e) Tan, Y.-H.; Li, J.-X.; Huo, J.-P.; Xue, F.-L.; Wang, Z.-Y. Synth. Commun. 2014, 44, 2974.

(f) Peng, P.; Feng, M.-H.; Shi, J.; Zheng, J.-L.; Wu, Y.-C.; Wang, Z.-Y.; Chen, R.-H. Lett. Org. Chem. 2015, 12, 359.

(Li, L.; Fan, Y.) 